\title{
The sequential judgment of proportion ${ }^{1,2}$
}

GORDON F. PITZ

SOUTHERN ILLINOIS UNIVERSITY

Ss gave percentage estimates of proportion, which were revised after each event during the presentation of a sequence of binary events. Responses exhibited constant errors of overestimation of proportions greater than .5 and underestimation of proportions less than .5. Both verbal and nonverbal responses were used, the constant error being somewhat greater for nonverbal responses. There was a tendency for the constant error to decrease as the number of events between revisions was increased.

In a sequential decision making task, a $\mathrm{S}$ must make a continuous re-evaluation of perceived events as they occur. One way of assessing the accuracy of this re-evaluation process is to have Ss make estimates of the proportion of times that some event has occurred. Constant errors in the percentage estimation of proportion have been reported by Shuford (1961), Erlick (1964), and Pitz (1965b), amongothers. The form of the constant error, however, has varied from one study to another. Both Erlick and Pitz used as stimuli sequences of binary events, with a single percentage estimate being given at the end of each sequence. The present study used similar sequences, but Ss were asked to make a revised percentage judgment after each event within the sequence. Sequential judgments were compared with estimates given only at the end of each sequence, and with judgments revised after differing numbers of events.

Method

The stimuli consisted of sequences of letters, 'B' or $'{ }^{\prime}$ ', presented with a stimulus duration of $0.5 \mathrm{sec}$, and an interstimulus interval of $0.2 \mathrm{sec}$. The letters

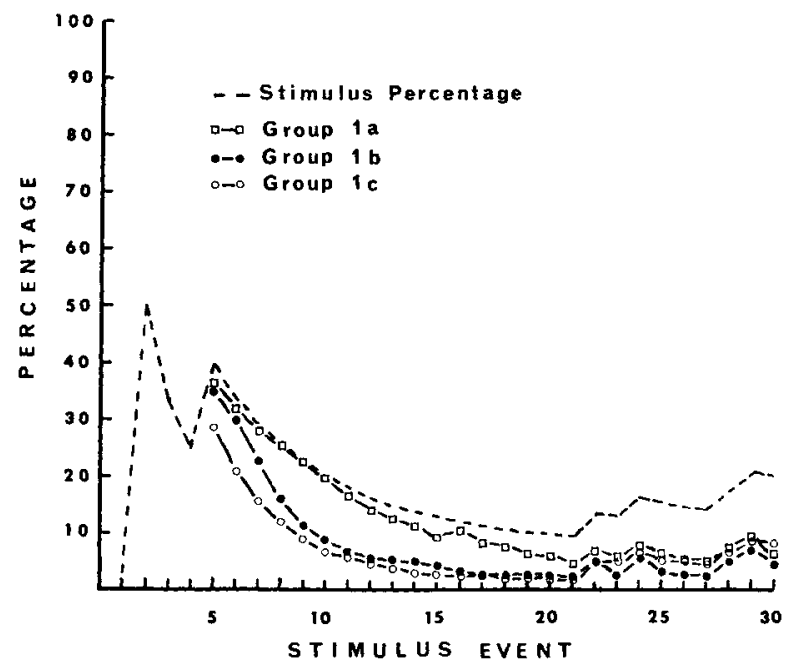

Fig. 1. Mean judgments by Groups $1 \mathrm{a}, 1 \mathrm{~b}$, and $1 \mathrm{c}$ to sequence 1 . were presented on an Industrial Electronics series 10,000 digital readout. All Ss were asked to estimate the percentage of ' $B$ 's during a sequence. Six different sequences of 30 letters were selected; the selection was not random, but rather was designed to provide three sequences of roughly constant proportions, and three of markedly changing proportions. Figure 1 shows one of the former sequences, and Fig. 2 one of the latter.

The study was run in two stages. In stage 1 , verbal percentage estimates were compared with nonverbal, scale-and-pointer responses. Three groups of $10 \mathrm{Ss}$ were used: Group 1a made judgments by means of verbal percentage estimates; Group $1 \mathrm{~b}$ used a scale, marked at the zero, 50 and 100 per cent points only, with a pointer which was left where set between responses; Group 1c used the same scale, but returned the pointer to the 50 per cent point between responses. A control group, 1d, of $10 \mathrm{Ss}$, was run to determine to what extent there were constant errors in the perception of percentage distances along the scale. This group was given values of percentages, and was asked to set the pointer to indicate these percentages on the scale. In stage 2, all Ss used the same scale and pointer device as used by Group 1b. Five groups of 10 Ss were used, Groups $2 \mathrm{a}$ through $2 \mathrm{e}$. The five groups were shown differing numbers of events between judgments: $1,2,5$, 10 , and 30 respectively, for sequences of 30 events. Results

A convenient means of summarizing the results is to fit to the data from each $\mathrm{S}$ a linear function relating the logistic transformation of the stimulus and estimated percentages, i.e.:

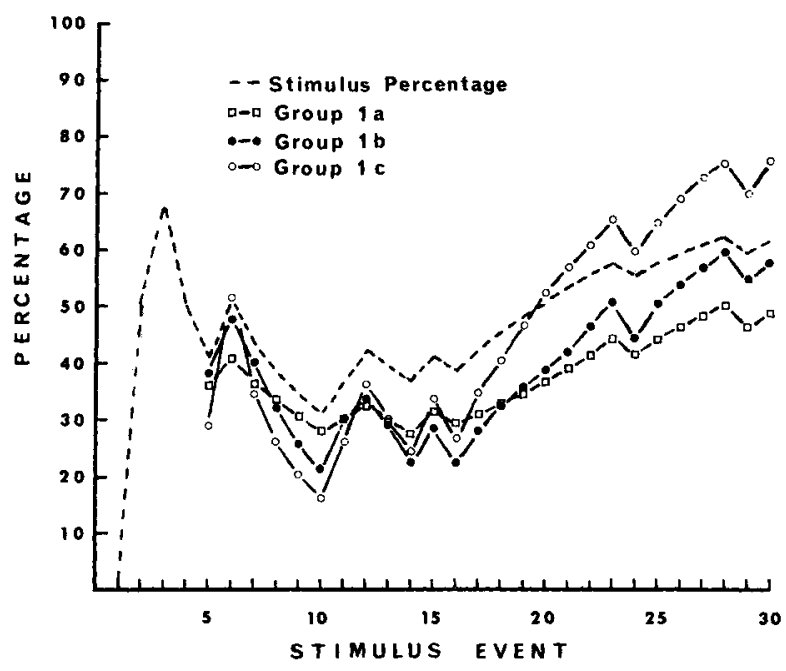

Fig. 2. Mean judgments by Groups 1a, 1b, and 1c to sequence 2 . 


$$
\log [\hat{\mathrm{P}} /(100-\hat{\mathrm{P}})]=\mathrm{a} \log [\mathrm{P} /(100-\mathrm{P})]+\mathrm{b}
$$

where $P$ is the stimulus percentage, and $P$ is the estimated percentage. This function is equivalent to an assumption that a power function, with exponent $a$, will describe the perception of ratios (see Pitz, 1965b). As an indication of the goodness of fit of this function, the average value of $\mathrm{r}^{2}$ for individual $\mathrm{Ss}$ in this study was .85 . The value of a gives an indication of the degree of constant error; if a is greater than 1, percentages greater than $50 \%$ are overestimated, and percentages less than $50 \%$ are underestimated. The mean value of a for Group 1a was 1.33, for Group $1 \mathrm{~b}$ was 1.71, and for Group $1 \mathrm{c}$ was 1.72 . All these means were significantly $(p=.01)$ greater than 1 . The degree of constant error was significantly larger for the two nonverbal groups than it was for the verbal group. Examination of mean responses by the control group, $1 d$, indicated no consistent or significant constant errors in placing the pointer to indicate specified percentages.

Figure 1 shows the average responses by Groups $1 \mathrm{a}, 1 \mathrm{~b}$, and $1 \mathrm{c}$ to one of the sequences of fairly constant stimulus percentage. Judgments for the first four events are not shown; they were almost invariably accurate. The form and magnitude of the constant error after event 4 is quite apparent. Figure 2 shows responses to one of the sequences of changing proportion. It is clear from this figure that there were sequential effects to be found, as well as the overall constant error. Judgments exhibited a lag in change as the stimulus percentage changed towards 50 per cent. The lag was greatest for Group la, and was very slight for Group 1c. These differences between groups were consistent over the three sequences of changing proportion, and showed up as significant Group by Event-Number interaction effects in analyses of variance for these sequences. This same lag in judgments can be seen to some extent in responses to the last 10 events of the sequence in Fig. 1.

Mean values of a for Groups $2 \mathrm{a}$ through $2 \mathrm{e}$ were $1.51,1.60,1.17,1.29$, and 1.40 respectively. Analysis of variance indicated a significant Groups effect $(p=.01)$, but no pairwise comparison, using a Tukey (a) test (Winer, 1962) was significant at that level. All exponents except that for Group 2c were significantly greater than 1.

\section{Discussion}

Although Erlick (1964) found that in the percentage estimation of proportion, judgments tended to be too close to 50 per cent, Pitz (1965b) found a slight tendency towards the opposite kind of error, associated with exponents a of greater than 1. Both of these studies used non-sequential judgments; in the present study, sequential judgments exhibited a pronounced tendency to be too extreme. Furthermore, with the use of nonverbal responses, the amount of error was even greater. Pitz (1965b) suggested that such a constant error may indicate a contrast effect occurring when a $S$ is able easily to discriminate the two events occurring in a binary sequence. It is reasonable to assume that in giving his judgments sequentially, a $S$ finds it easier to perceive the occurrence of ' $B$ 's separately from the occurrence of 'C's. Some support for this argument is to be found in the general tendency for constant errors, and possible contrast effects, to decrease as the number of events between judgments increased.

The reason for the discrepancy between verbal and nonverbal responses is not clear. However, Pitz (1965a) showed that the use of verbal-numerical responses in a magnitude estimation task induced irregularities in the resultant psychophysical scale, which appeared to be due to the existence of biases in the use of numbers. These irregularities did not appear when a nonverbal, magnitude production method was used. It is entirely possible, of course, that nonverbal response devices produce artifacts of their own, which affected responses in the present study.

The lag in judgments which is illustrated in Fig. 2 was apparently a function of a S's recall of his previous response.Group 1b left the pointer where it had been set between responses, and hence could see their previous response, while Group 1a could presumably recall their previous verbal response. These two groups exhibited the lag far more than did Group 1c, who returned the pointer to the 50 per cent position between responses.

\section{References}

Erlick, D. E. Absolute judgments of discrete quantities randomly distributed over time. J. exp. Psychol, 1964, 67, 475-482.

Pitz, G. F. Magnitude scales of line length. Psychon. Sci, 1965a, 2, 213-214.

Pitz, G. F. Response variables in the estimation of relative frequency. Percept. mot. Skills, 1965b, 21, 867-873.

Shuford, E. H. Percentage estimation of proportion as a function of element type, exposure time, and task. J. exp. Psychol., $1961,61,430-436$.

Winer, B. J. Statistical principles in experimental design. New York: McGraw-Hill, 1962.

\section{Notes}

1. This research was supported in part by grant DMH 17-184 from the Psychiatric Training and Research Fund of the Illinois Department of Mental Health, and in part by grant MH 10167-01 from the National Institute of Mental Health, U. S. Public Health Service.

2. Considerable assistance in the running of this experiment was supplied by Darrell Willis, to whom the author is very grateful. 